\title{
Análise da superfície de grês porcelanato por microscopia de força atômica
}

\section{(Porcelain stoneware tile surface analysis by atomic force microscopy)}

\author{
M. C. S. Fernandes, P. I. Paulin Filho, M. R. Morelli \\ Departamento de Engenharia de Materiais - UFSCar, Rod. Washington Luiz km 235, S. Carlos, SP 13565-905 \\ merilin_csf@yahoo.com.br,paulin@ufscar.br,morelli@ufscar.br
}

\begin{abstract}
Resumo
A superfície de porcelanatos polidos foi investigada através da Microscopia de Força Atômica (AFM). Ênfase foi dada à investigação dos parâmetros de rugosidade e brilho que estão relacionados com a evolução do processo de polimento da superfície do porcelanato. A rugosidade da superfície é uma característica que interfere em diversas propriedades de interesse do produto, às vezes negativamente, sendo interessante a aplicação de resinas poliméricas que tendem a nivelar as irregularidades superficiais. Neste sentido, devido à possibilidade de obtenção de imagens tridimensionais no AFM, foi possível realizar medidas topográficas para a obtenção dos principais parâmetros que interferem no acabamento superficial do porcelanato, de forma a contribuir para um melhor entendimento do processo de polimento nas características estéticas do produto. Os resultados obtidos revelaram que a diminuição da rugosidade ocorreu mais visivelmente nas etapas de polimento com abrasivos de grãos maiores, passando de 0,650 $\mu \mathrm{m}$ a 0,192 $\mu \mathrm{m}$ entre os estágios de grit 80 e 1000 na área de varredura de 80 x $80 \mu \mathrm{m}^{2}$, enquanto os abrasivos constituídos de grãos menores interferiram pouco na rugosidade superficial, sendo os principais responsáveis pelo brilho final das peças, cujo valor aumentou de 29,3 a 42,2 UB (unidades de brilho) entre os estágios de grit 800 e 1500. O recobrimento da superfície com a resina polimérica reduziu significativamente o brilho do produto, sem causar alterações bruscas na rugosidade superficial.
\end{abstract}

Palavras-chave: porcelanato, polimento, AFM, rugosidade, brilho.

\begin{abstract}
The surface of polished porcelain stoneware tile surface was investigated through Atomic Force Microscopy (AFM). Emphasis was given to the investigation of the roughness and gloss parameters which are related with the polishing process evolution of the porcelain stoneware tile surface. The surface roughness is a characteristic that intervenes in different interest properties of the product, sometimes negative, being interesting the application of the polymeric resins that tends to level the surface irregularities. In this way, due to possibility of three-dimensional images attainment in AFM, topographical measures was made for the attainment to the main parameters that intervenes in finishing of porcelain stoneware tile surface, on form to contribute for a better agreement of the polishing process in the aesthetic product characteristics. The results achieved disclose that the roughness reduction occurred more visibly in the polishing stages with larger grains, going from $0.650 \mu \mathrm{m}$ to $0.192 \mu \mathrm{m}$ between the stages of grit 80 and 1000 in the scanning area of $80 \times 80 \mathrm{\mu m}^{2}$, while the abrasives consisted of smaller grains intervene poorly in superficial roughness, being the main responsible for the samples final gloss, whose value increase from 29.3 to $42.2 \mathrm{GU}$ (gloss units) between the stages of grit 800 and 1500. The surface covering with the polymeric resin reduced significantly the product gloss, without it causes brusque alterations in superficial roughness.
\end{abstract}

Keywords: porcelain stoneware tile, polishing, AFM, roughness, gloss.

\section{INTRODUÇÃO}

\section{O porcelanato como objeto de estudo}

O Brasil é um dos principais protagonistas no mercado mundial de revestimentos cerâmicos porque possui o segundo maior mercado consumidor, é o quinto maior exportador e ocupa a segunda posição no ranking mundial de produtores [1]. O porcelanato é um dos produtos que apresentam grande expansão na escala produtiva [2]. Em 1999 foram produzidos no Brasil cerca de 3,4 milhões de $\mathrm{m}^{2}$ [3]. Em 2008, a produção alcançou 46 milhões de $\mathrm{m}^{2}$ [1].

O porcelanato é um derivado da porcelana. A principal diferença entre a porcelana e o porcelanato está no programa de queima. Enquanto a porcelana é queimada durante um longo tempo (em torno de $24 \mathrm{~h}$ ), o porcelanato é fabricado com um ciclo de queima mais rápido (de 60 a $90 \mathrm{~min}$ ) [4]. Assim o termo porcelanato refere-se a uma cerâmica com massa extremamente compacta, cuja microestrutura apresenta uma fase cristalina imersa em uma matriz vítrea, sendo queimada a uma temperatura próxima de $1200{ }^{\circ} \mathrm{C}$ [2]. Possui excelentes propriedades e características como: baixa porosidade, baixa absorção de água, elevada dureza, elevada resistência química, elevada resistência à abrasão, elevada resistência mecânica, elevada resistência ao congelamento e características estéticas agradáveis [5-6].

Para melhorar o efeito estético e a posição competitiva com as pedras naturais, os porcelanatos são submetidos a um processo de polimento. Entretanto, tal processo pode ocasionar danos irreversíveis no produto, principalmente 
devido à abertura da porosidade fechada e à formação de defeitos superficiais que afetam diversas propriedades de interesse do produto. Desta forma, alguns fabricantes realizam o recobrimento da superfície, após o último polimento, com resinas que tendem a amenizar tais irregularidades. No entanto, essas resinas não possuem longa durabilidade, trazendo incômodo e desconfiança ao consumidor [5]. Assim, estudos sobre a rugosidade superficial de porcelanatos podem contribuir para um melhor entendimento e controle do processo responsável pelas características finais do produto.

\section{Rugosidade superficial dos porcelanatos}

A rugosidade superficial é definida como um conjunto de irregularidades, tais como saliências e reentrâncias, presentes em uma superfície [7]. Nos porcelanatos, a rugosidade superficial afeta as seguintes propriedades de interesse do produto [8]: propriedades óticas - o brilho e a nitidez da imagem refletida pelo produto são alterados pelas reentrâncias e saliências presentes em sua superfície. Com a redução da rugosidade, a nitidez da imagem refletida aumenta, aumentando o brilho; resistência química - elevada rugosidade superficial aumenta a área de contato do revestimento cerâmico com as soluções de ataque ou mesmo com os agentes atmosféricos, acelerando a deterioração superficial; resistência à abrasão - as irregularidades se desprendem com maior facilidade da superfície do revestimento cerâmico durante o desgaste, diminuindo a resistência à abrasão; resistência ao manchamento - as depressões podem reter sujeira difícil, ou até mesmo impossível, de remover. Assim, a resistência ao manchamento é menor em superfícies mais rugosas; resistência ao escorregamento - é maior em superfícies com rugosidades elevadas. Isso porque texturas rugosas aumentam o coeficiente de atrito superficial, já que há maior área de contato entre a superfície do revestimento e os calçados.

Desta forma, é possível concluir que o estudo da microestrutura da superfície fornece o embasamento necessário para o entendimento do comportamento do produto e sua possível melhora. Neste sentido, o AFM, além de fornecer imagens da topografia da superfície, permite a medida direta dos parâmetros de rugosidade.

\section{Vantagens da microscopia de força atômica}

O Microscópio de Força Atômica (AFM - Atomic Force Microscope) é um instrumento de imagem mecânico que mede a topografia das três dimensões, além das propriedades físicas da superfície de uma amostra, com uma sonda pontiaguda. É ideal para medir quantitativamente em escala nanométrica a rugosidade superficial e para visualizar a nanotextura de muitos tipos de superfícies de materiais, possuindo uma elevada resolução espacial tridimensional.

Segundo comparação de Bernardes Filho e Mattoso [9] o AFM trabalha de forma semelhante às agulhas dos antigos toca-discos, sendo que no lugar da agulha (probe), encontra-se o cantilever, que consiste de uma haste flexível com uma ponta de poucos mícrons na parte inferior. Para varrer a amostra e obter a imagem, é utilizado um sistema de posicionamento usando cerâmicas piezoelétricas, que percorrem a amostra nas três direções com precisão de ângstrons. Nesta varredura emprega-se também um sistema de alinhamento com feixe de laser que incide sobre o cantilever e reflete em um sensor de quatro quadrantes. Este sensor fornece informações de localização para o sistema de realimentação e controle, que corrige a posição do cantilever de forma a manter o contato com a amostra e permitir a obtenção da imagem. A Fig. 1 mostra o diagrama de funcionamento do AFM.

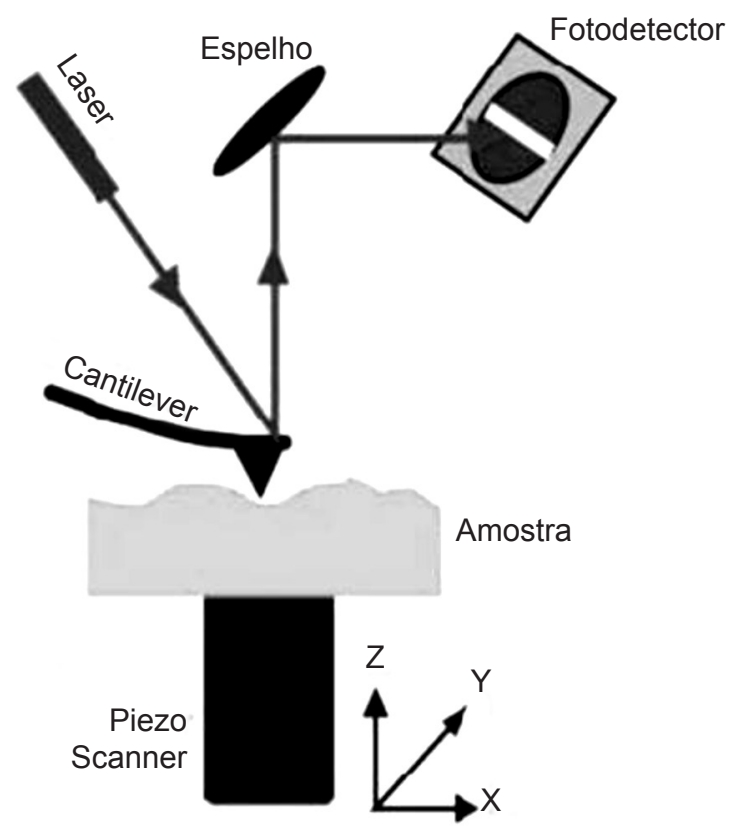

Figura 1: Diagrama de funcionamento do microscópio de força atômica.

[Figure 1: Operation diagram of an atomic force microscope.]

Os diferentes modos de se obter uma imagem se referem à distância mantida entre a sonda e a amostra no momento da varredura, e às formas de movimentar a ponteira sobre a superfície estudada [10]. Eles variam em função de diversos fatores como natureza da amostra, tipo de cantilever utilizado e o tipo de varredura [9]. Os modos de fazer imagens podem ser classificados em modo contato e modo não-contato, dependendo das forças líquidas entre a sonda e a amostra. Se o aparelho opera na região atrativa (onde cantilever e amostra são atraídos), trata-se do modo não-contato. Se a operação é na região repulsiva (onde cantilever e amostra são repelidos), trata-se do modo contato [10]. A atração e repulsão causam uma deflexão no cantilever, mostrada na Fig. 2.

Além das medidas topográficas é possível obter imagens com contraste que diferenciam os diferentes materiais de acordo com sua rigidez. Assim, a técnica de AFM pode diferenciar materiais mesmo que não haja diferença de topografia. Ela também permite o mapeamento de cargas 


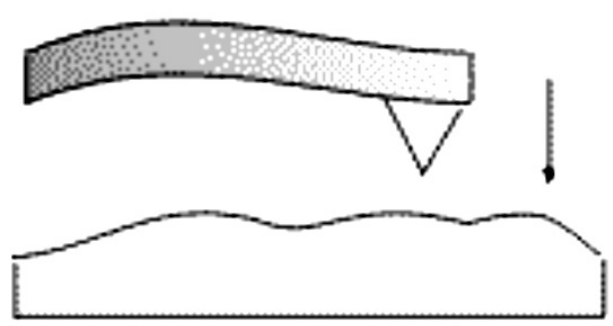

Não contato

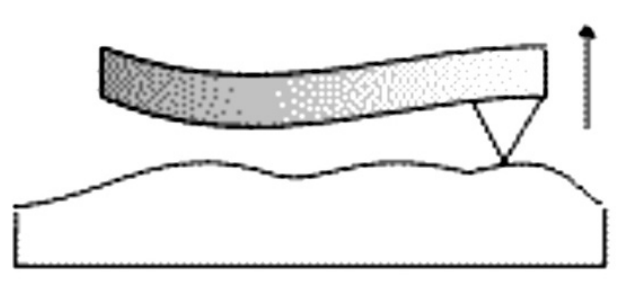

Contato

Figura 2: Deflexão do cantilever operando no modo não-contato e contato [10].

[Figure 2: Cantilever deflection operating in non-contact and contact mode.]

elétricas em superfícies carregadas, auxiliando o estudo de compósitos condutores elétricos, pois é possível visualizar e identificar as áreas que apresentam maior concentração de cargas. Nesta mesma linha, é possível realizar medidas de força magnética, o que permite mapear domínios magnéticos em superfícies magnetizadas [9].

\section{Brilho superficial}

Para melhorar as propriedades estéticas do porcelanato a etapa de polimento é essencial na garantia de um elevado brilho. Em revestimentos cerâmicos, brilho é a capacidade da superfície de refletir a luz incidente predominantemente numa mesma direção [11]. A reflexão da luz em diversas direções acontece no caso de superfícies rugosas, enquanto em superfícies lisas espera-se que a reflexão ocorra numa direção predominante. Superfícies lisas e com pouca absorção de luz são consideradas formadoras de imagens [11]. O brilho superficial é mais uma impressão subjetiva do observador do que uma propriedade física. Entretanto, a parte especular do brilho pode ser medida objetivamente através do equipamento conhecido por brilhômetro. Este equipamento é utilizado devido à praticidade e rapidez com que fornece os resultados. Neste trabalho foi observado e analisado o ganho de brilho ao longo do processo de polimento dos porcelanatos e sua relação com a rugosidade superficial.

\section{MATERIAIS E MÉTODOS}

\section{Materiais e sua preparação}

Foram coletadas peças de porcelanatos técnicos comerciais de uma empresa que representa significativamente o setor brasileiro de revestimentos cerâmicos. As peças foram coletadas durante os estágios de polimento pelos quais passam os produtos. As amostras foram analisadas conforme recebidas, não sendo realizado qualquer processo adicional de tratamento superficial. Os revestimentos cerâmicos com dimensões industriais foram cortados em equipamentos de corte com discos diamantados nas dimensões de $10 \mathrm{~cm} \times 10 \mathrm{~cm}$, para determinação do brilho, e $10 \mathrm{~mm}$ x $10 \mathrm{~mm}$ x $2 \mathrm{~mm}$, para análise no AFM. Em seguida passaram apenas por um processo de limpeza com detergente neutro.

\section{Obtenção de imagens no AFM}

O AFM empregado neste estudo foi um microscópio MultiMode $^{\text {TM }}$ IIIa SPM da Digital Instruments (USA). Este equipamento opera com uma sonda pontiaguda de nitreto de silício do tipo NP em forma de V, da Digital Instr., conforme Fig. 3.

O modo de varredura utilizado foi o de contato, no qual a ponteira toca suave e constantemente a amostra. Desta forma as forças repulsivas dominam a interação entre ponteira e amostra. Esta forma de obtenção de imagens é mais indicada para amostras rígidas, pois a amostra não é danificada pela varredura do cantilever. As imagens foram obtidas para três áreas diferentes de varredura: $2 \times 2 \mu \mathrm{m}^{2}, 10 \times 10 \mu \mathrm{m}^{2}$ e 80 $\times 80 \mu^{2}$. A primeira varredura foi feita para áreas maiores $\left(80 \times 80 \mu \mathrm{m}^{2}\right)$ sendo então reduzida até que áreas menores $\left(2 \times 2 \mu \mathrm{m}^{2}\right)$ fossem alcançadas. Cada medida foi realizada três vezes para a obtenção de um valor médio de rugosidade.

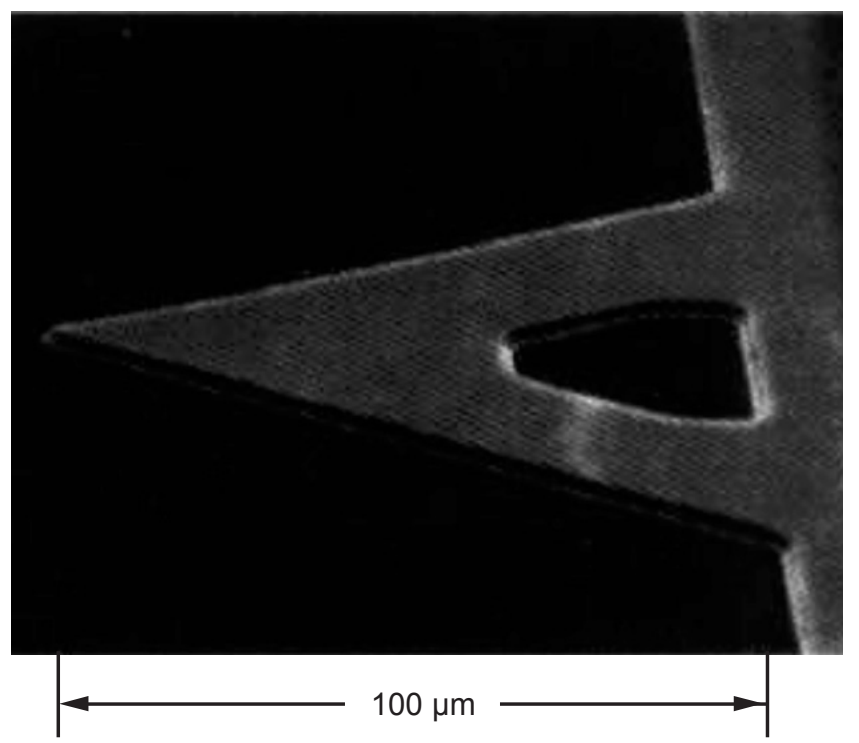

Figura 3: Cantilever em forma de V [10]. [Figure 3: Cantilever in Vform.]

\section{Tratamentos das imagens}

O software de tratamento de imagem da NanoScope $\AA$ contém algoritmos poderosos para apresentação e medição dos resultados. As imagens realizadas podem ser observadas em duas e três dimensões, podendo também visualizar-se 
secções de corte, efetuar a medição da rugosidade superficial, realizar análise de perfil de profundidade, entre outros. A rugosidade média $(\mathrm{Ra})$ é calculada pelo programa através da média aritmética dos valores absolutos das alturas dos pontos que compõem o perfil, em relação à linha média (LM). A linha média é uma linha de referência que divide o perfil de rugosidade, de forma que a soma das áreas superiores seja igual à soma das áreas inferiores. O parâmetro Ra é o mais utilizado na indicação da rugosidade superficial. Vale ressaltar, no entanto, que ele apresenta baixa sensibilidade para indicar a presença de picos e vales ao longo dos perfis rugosimétricos. Dessa forma, foi possível medir diretamente a rugosidade $\mathrm{Ra}$ dos porcelanatos nas diferentes etapas do processo de polimento e estudar a influência do tamanho da área de varredura nos valores de rugosidade obtidos.

\section{Medida de brilho}

Os valores de brilho foram medidos ao longo de toda a superfície das peças de porcelanato com um brilhômetro Horiba IG-320, com ângulo de incidência $60^{\circ}$. Foram feitas cinco medidas em cada peça para a obtenção do valor médio de brilho. Os valores de brilho obtidos foram comparados com as rugosidades superficiais correspondentes a cada etapa de polimento.

\section{RESULTADOS E DISCUSSÃO}

Os estágios de polimento são diferenciados pelo tamanho médio do abrasivo utilizado, que é sucessivamente menor de um estágio para o próximo. No caso do porcelanato investigado o tamanho dos grãos variou de 80 a 1500 mesh, conforme Tabela I. Após o polimento com abrasivo de grit 1500 o produto passou por uma impermeabilização da superfície com resina polimérica, a fim de preencher os poros abertos e nivelar as irregularidades superficiais. Tal estágio foi igualmente estudado neste trabalho.

Tabela I - Tamanho médio do abrasivo. [Table I - Abrasive average size.]

\begin{tabular}{cc}
\hline grão $($ grit $)$ & Tamanho médio $(\mu \mathrm{m})$ \\
\hline 80 & 185,0 \\
120 & 109,0 \\
180 & 69,0 \\
320 & 29,2 \\
800 & 6,5 \\
1000 & 4,5 \\
1200 & 3,0 \\
1500 & 2,0 \\
\hline
\end{tabular}

A superfície do porcelanato polido foi examinada pelo AFM em cada estágio de polimento pelo qual passa o produto. A característica das superfícies foi estudada em termos de rugosidade superficial. As Figs. 4 e 5 mostram a diferença nas morfologias da superfície obtidas pelas topografias bi e tridimensionais da amostra polida com o abrasivo de grit 1500 e a amostra recoberta com resina polimérica, respectivamente. Para cada amostra foram obtidas imagens com três diferentes áreas de varredura $(2 \times$ $2 \mu \mathrm{m}^{2}, 10 \times 10 \mu \mathrm{m}^{2}$ e $\left.80 \times 80 \mu \mathrm{m}^{2}\right)$.

Nas imagens, as áreas mais escuras correspondem às regiões mais baixas (reentrâncias) da superfície das amostras, enquanto as áreas mais claras mostram as regiões mais salientes. É possível observar pelas Figs. 4 e 5 que a área de varredura de $80 \times 80 \mu \mathrm{m}^{2}$ fornece uma visão mais geral da superfície da amostra, já que abrange uma área maior. Conforme a área de varredura vai diminuindo passa-se a ter uma visão mais localizada de alguns pontos específicos da amostra e a análise se torna menos reprodutível.

De acordo com a Fig. 4, no último estágio de polimento (grit 1500) a superfície do porcelanato apresenta ainda algumas saliências e poros abertos. Além disso, nas Figs. $4 \mathrm{~b}, 4 \mathrm{c}, 4 \mathrm{e}$ e $4 \mathrm{f}$ são observados alguns riscos profundos que decorrem provavelmente do processo de polimento, já que o movimento das politrizes pode fazer com que alguns pontos sejam mais desgastados que outros, provocando os riscos observados.

Na Fig. 5, correspondente à superfície polida com abrasivo de grit 1500 e recoberta com resina polimérica, as irregularidades da superfície são menos visíveis, mas alguns poros ainda podem ser observados. Isso não significa que o processo de impermeabilização seja ineficiente para alcançar o nivelamento da superfície, mas que esta área especificamente analisada possuía poros de profundidade diferente dos analisados na Fig. 4. Para uma análise em que se visualize melhor a eficiência do processo de impermeabilização, a mesma região de uma mesma amostra deveria ser observada antes e depois da aplicação da resina.

As Figs. 6,7 e 8 mostram os gráficos de evolução da rugosidade ao longo do processo de polimento da superfície do porcelanato. Tais figuras mostram, respectivamente, os valores de rugosidade média $\mathrm{Ra}$ medidos nas áreas de varredura de $80 \times 80 \mu \mathrm{m}^{2}, 10 \times 10 \mu \mathrm{m}^{2} \mathrm{e} 2 \times 2 \mu \mathrm{m}^{2}$. As barras verticais indicam o desvio padrão encontrado nas medições.

A partir dos resultados apresentados nas Figs. 6, 7 e 8 , nota-se que quanto maior a área de varredura, maior a rugosidade. $\mathrm{O}$ aumento da rugosidade junto com o aumento da área de varredura pode estar relacionado à dependência da rugosidade com a freqüência da área de varredura [12]. Para uma área superficial pequena, somente a rugosidade das freqüências "mais altas" é medida. Quando uma maior área superficial é varrida, a rugosidade causada por freqüências adicionais mais baixas têm que ser levadas em consideração, resultando em valores mais altos quando uma área superficial maior é varrida. Desta forma, quando o tamanho da varredura é mudado, uma topografia de superfície diferente é obtida, levando a em diferentes rugosidades. Assim, é crucial que a mesma escala do tamanho de varredura seja usada ao comparar a rugosidade superficial de amostras diferentes.

Como a área de varredura de $80 \times 80 \mu \mathrm{m}^{2}$ foi a mais reprodutível, já que apresentou menores valores de desvio padrão, ela foi escolhida para correlacionar a rugosidade 

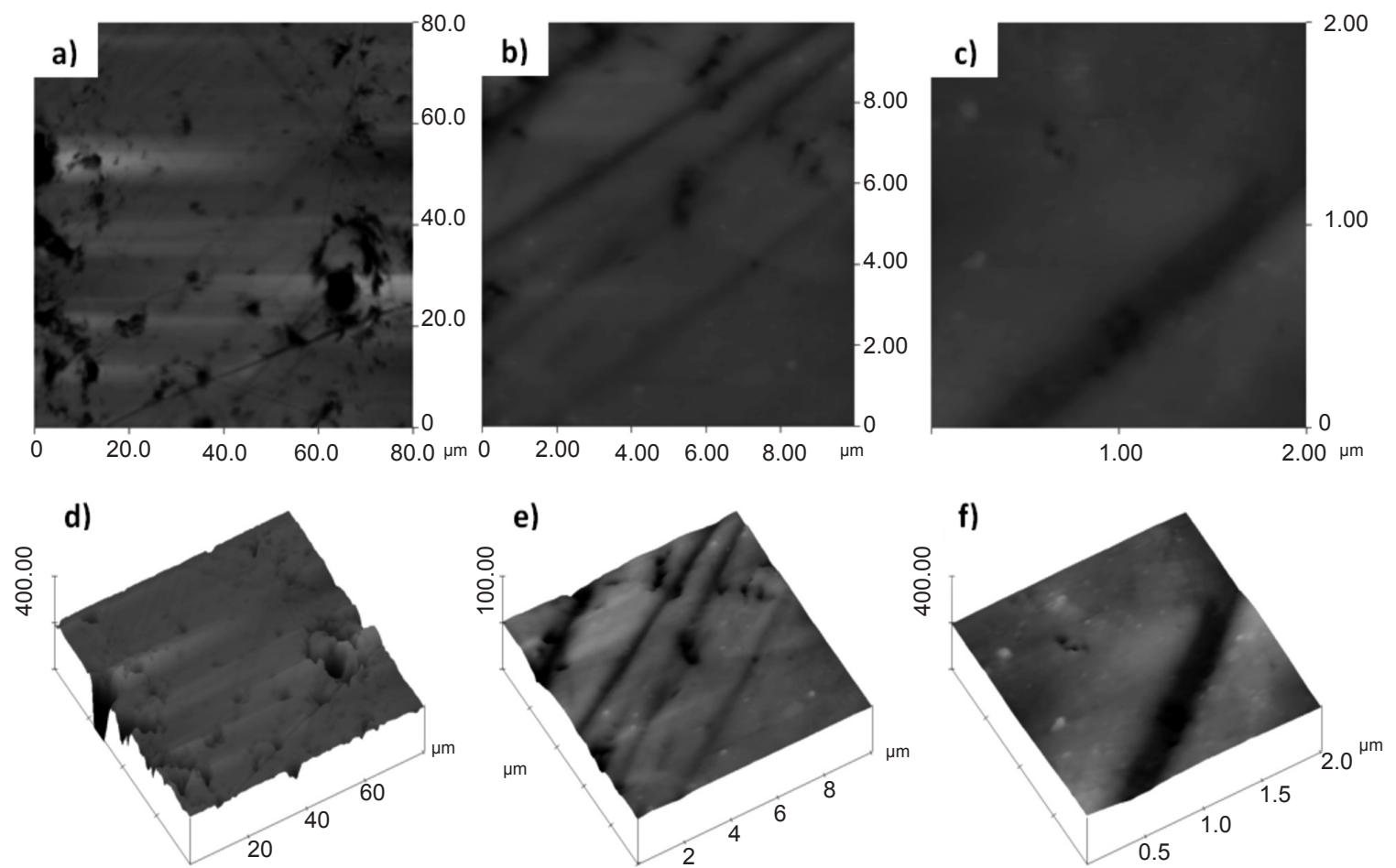

Figura 4: Imagens de topografia bi (a, b, c) e tridimensionais (d, e,f) da amostra de porcelanato polida com abrasivo de grit 1500. a, d) Área de varredura de $80 \times 80 \mu \mathrm{m}^{2}$. b, e) Área de varredura de $10 \times 10 \mu \mathrm{m}^{2}$. c, f) Área de varredura de $2 \times 2 \mu \mathrm{m}^{2}$.

[Figure 4: Two $(a, b, c)$ and three-dimensional $(d, e, f)$ topography images of porcelain stoneware tile sample polishing with abrasive of grit 1500. a, d) Scanning area of $\left.80 \times 80 \mu^{2} . b, e\right)$ Scanning area of $\left.10 \times 10 \mu \mathrm{m}^{2} . c, f\right)$ Scanning area of $\left.2 \times 2 \mu \mathrm{m}^{2}.\right]$
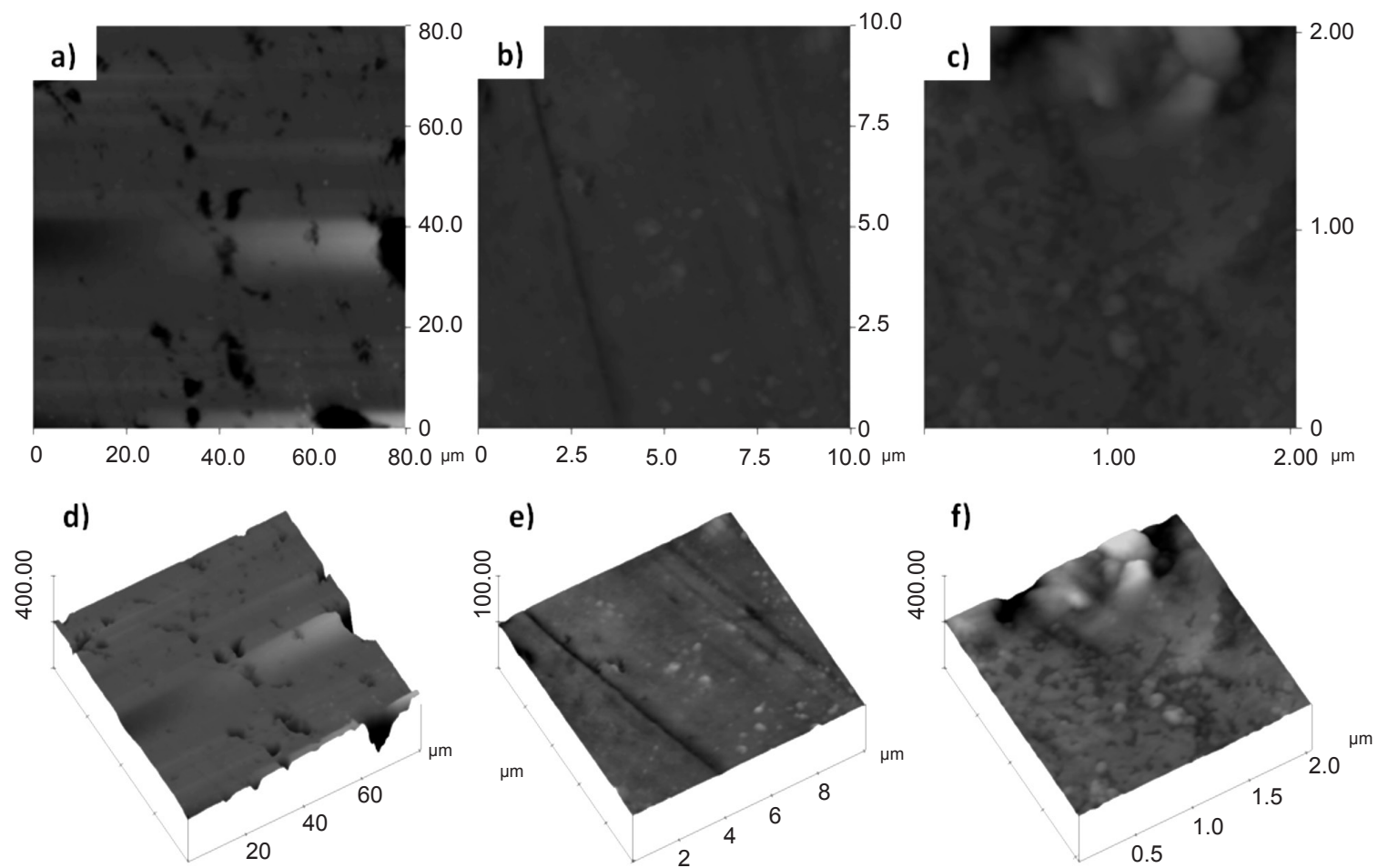

Figura 5: Imagens de topografia bi $(\mathrm{a}, \mathrm{b}, \mathrm{c})$ e tridimensionais $(\mathrm{d}, \mathrm{e}, \mathrm{f})$ da amostra de porcelanato recoberta com resina polimérica. a, d) Área de varredura de $80 \times 80 \mu \mathrm{m}^{2}$. b, e) Área de varredura de $10 \times 10 \mu \mathrm{m}^{2}$. c, f) Área de varredura de $2 \times 2 \mu \mathrm{m}^{2}$.

[Figure 5: Two $(a, b, c)$ and three-dimensional $(d, e, f)$ topography images of porcelain stoneware tile sample recovering with polymeric resin. a, d) Scanning area of $\left.80 \times 80 \mu \mathrm{m}^{2} . b, e\right)$ Scanning area of $10 \times 10 \mu \mathrm{m}^{2} . c, f$ ) Scanning area of $\left.2 \times 2 \mu \mathrm{m}^{2}.\right]$ 


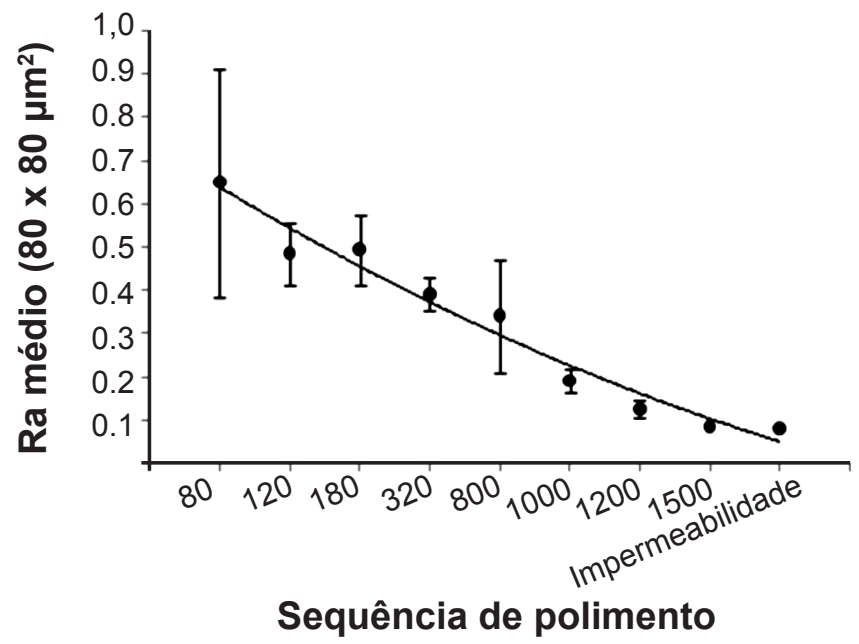

Figura 6: Evolução do processo de polimento da superfície do porcelanato, obtida por AFM, em função da rugosidade para a área de varredura de $80 \times 80 \mu \mathrm{m}^{2}$.

[Figure 6: Evolution of porcelain stoneware tile surface polishing process, attainment for AFM, in function of roughness for the scanning area of $80 \times 80 \mu^{2}$.]

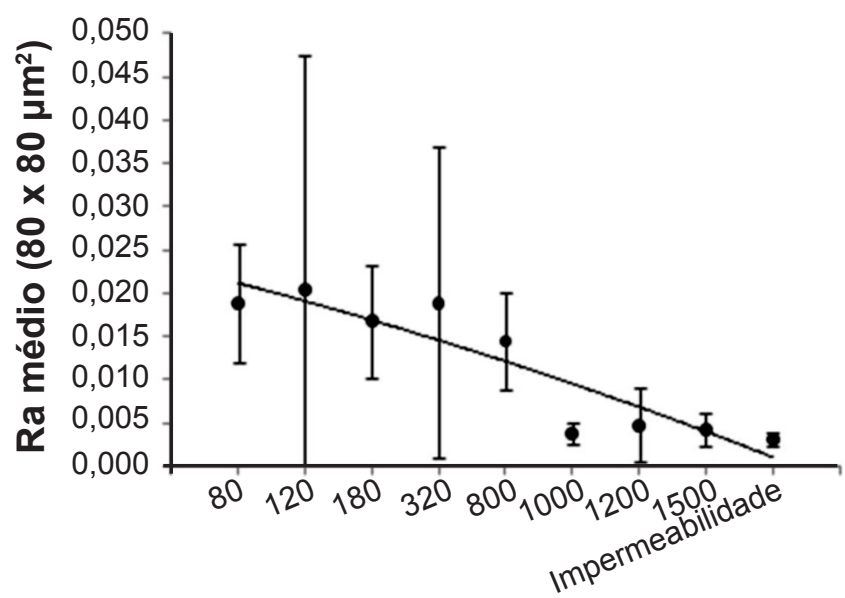

Sequência de polimento

Figura 8: Evolução do processo de polimento da superfície do porcelanato, obtida por AFM, em função da rugosidade para a área de varredura de $2 \times 2 \mu \mathrm{m}^{2}$.

[Figure 8: Evolution of porcelain stoneware tile surface polishing process, attainment for AFM, in function of roughness for the scanning area of $2 \times 2 \mu \mathrm{m}^{2}$.]

superficial e o brilho das amostras de porcelanato (Fig. 9).

$\mathrm{Na}$ Fig. 9 pode ser observado que a rugosidade superficial é gradativamente menor ao longo da evolução do processo de polimento. Observa-se que do primeiro para o segundo estágio de polimento, que possuem tamanho médio de abrasivo de respectivamente 185,0 e 109,0 $\mu \mathrm{m}$ (grit 80 e 120), houve uma queda brusca do valor da rugosidade superficial média, de 0,650 a $0,486 \mu \mathrm{m}$. Após a etapa de polimento com grit 120 a diminuição da rugosidade superficial deu-se de forma suave até o grit 800 ser alcançado. Nestes primeiros estágios de polimento iniciou-se a retirada dos sulcos, preparando a superfície para os abrasivos de menor granulometria que

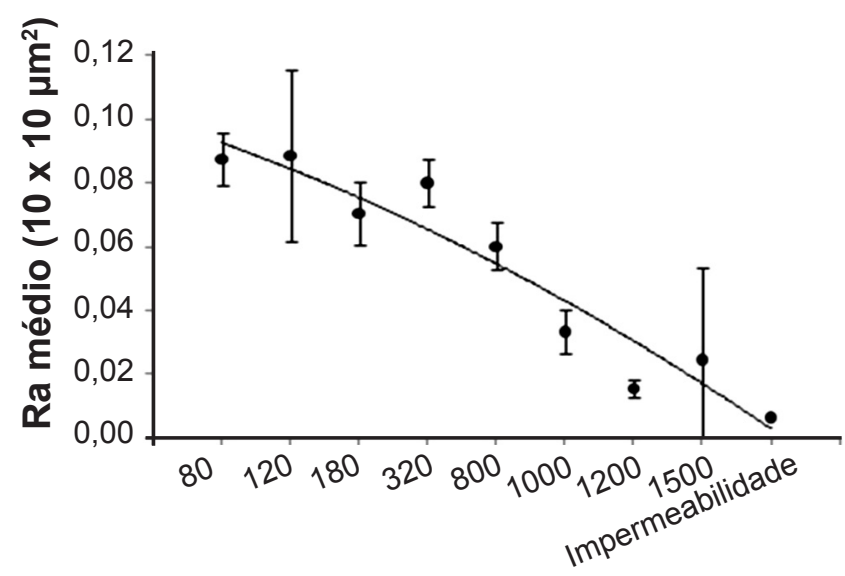

Sequência de polimento

Figura 7: Evolução do processo de polimento da superfície do porcelanato, obtida por AFM, em função da rugosidade para a área de varredura de $10 \times 10 \mu \mathrm{m}^{2}$.

[Figure 7: Evolution of porcelain stoneware tile surface polishing process, attainment for AFM, as a function of roughness for the scanning area of $10 \times 10 \mu^{2}$.]

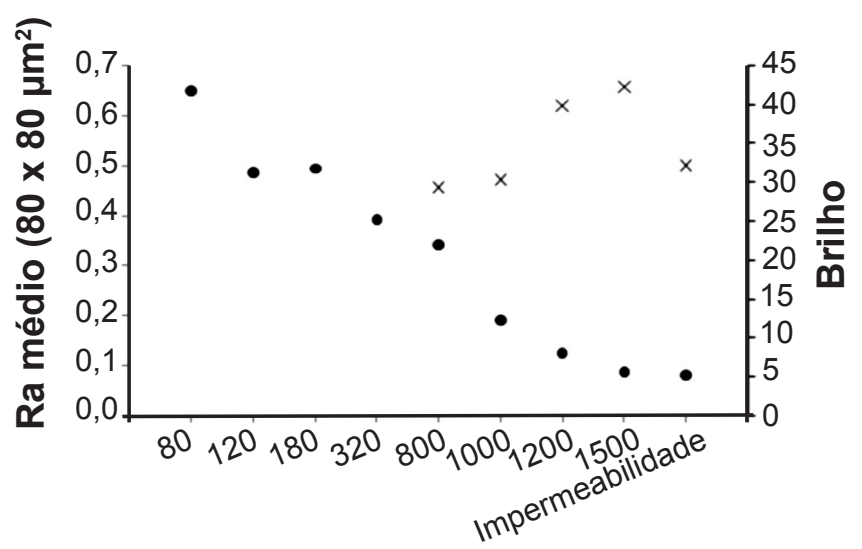

\section{Sequência de polimento}

Figura 9: Correlação entre rugosidade superficial e brilho de superfícies de porcelanato a partir de medidas por AFM e brilhômetro com ângulo de incidência de $60^{\circ}$.

[Figure 9: Correlation between superficial roughness and gloss of porcelain stoneware tile surface from AFM measures and glossmeter with incidence angle of $60^{\circ}$.]

provocam o aumento substancial no brilho das peças. Os valores de brilho foram obtidos apenas a partir do estágio em que se utilizaram abrasivos de grit 800 já que abaixo deste valor o brilho da superfície é insignificante. A partir do estágio com grit 800 houve nova queda significativa na rugosidade superficial, em torno de 0,149 $\mu \mathrm{m}$. Esta diminuição de rugosidade provocou um aumento de um ponto no valor brilho, o que não foi suficiente para fornecer o brilho desejável ao produto. A próxima etapa de polimento reduziu a rugosidade em apenas $0,066 \mu \mathrm{m}$, mas provocou um aumento brusco de brilho, que de 30,3 UB passou para 39,8 UB. Nos estágios com grit 1200 e 1500 não houve 
aumento significativo da rugosidade, entretanto, o brilho alcançou seus maiores valores nestes estágios. É possível observar então que no estágio final de polimento os valores de brilho praticamente dobram, quando comparados com os valores iniciais obtidos.

A aplicação da resina polimérica na superfície do porcelanato não alterou de forma significativa sua rugosidade, mas causou uma elevada diminuição no valor de brilho (32,2 UB), chegando quase ao valor inicial obtido (29,3 UB). De modo geral, os abrasivos com grãos maiores foram responsáveis pela diminuição acentuada da rugosidade superficial, enquanto os abrasivos com grãos menores forneceram o brilho final das peças.

\section{CONCLUSÕES}

O processo de polimento agrega valores ao porcelanato técnico devido a sua capacidade de melhorar a qualidade estética do revestimento cerâmico. Analisando-se os parâmetros de rugosidade superficial e brilho ao longo do polimento é possível compreender o processo que garante a satisfação do consumidor ao adquirir tal produto. Neste sentido, a técnica de AFM é uma ferramenta poderosa de observação e medição de irregularidades superficiais, dispondo de várias formas de obtenção de imagens e parâmetros. Entretanto, para obter valores comparáveis de algumas características em diferentes amostras, como a rugosidade superficial, é necessário que as análises sejam realizadas com a mesma área de varredura. Os resultados mostraram que a diminuição da rugosidade superficial foi mais acentuada entre os estágios de grit 80 e 1000 , passando de $0,650 \mu \mathrm{m}$ a $0,192 \mu \mathrm{m}$ na área de varredura de 80 x $80 \mu \mathrm{m}^{2}$. $\mathrm{O}$ brilho do produto praticamente dobrou entre o primeiro estágio analisado $(800 \mu \mathrm{m})$ e o último $(1500 \mu \mathrm{m})$ aumentando do valor inicial de 29,3 UB a 42,2 UB. Desta forma é possível concluir que a diminuição da rugosidade ocorreu mais visivelmente nas etapas de polimento com abrasivos de grãos maiores, enquanto os abrasivos constituídos de grãos menores foram os responsáveis pelo brilho final das peças, interferindo pouco na rugosidade superficial. A impermeabilização da superfície causou uma diminuição brusca do brilho, sem alteração significativa da rugosidade.

\section{AGRADECIMENTOS}

Aos Srs. Celso e Milton da empresa Villagres pelas medidas de brilho e à CAPES e PPG-CEM/UFSCar pelo incentivo à pesquisa.

\section{REFERÊNCIAS}

[1] ANFACER - Associação Nacional dos Fabricantes de Cerâmica de Revestimento, disponível em http://www. anfacer.org.br/, 04/01/2010.

[2] H. J. Alves, F. B. Minussi, F. G. Melchiades, A. O. Boschi, Ceram. Ind. 14, 1 (2009) 21-26.

[3] A. P. M. Menegazzo, F. L. N. Lemos, J. O. A. Paschoal, D. Gouvêa, J. C. Carvalho, R. S. N. Nóbrega, Ceram. Ind. 5, 5 (200) 7-10.

[4] J. Martín-Márquez, J. M. Rincón, M. Romero, Ceram. Int. 34 (2008) 1867-1873.

[5] P. M. T. Cavalcante, M. Dondi, E. R. Almendra, G. Ercolani, G. Guarini, M. Raimondo, Ceram. Ind. 9, 5/6 (2004) 29-41.

[6] M. Raimondo, M. Zanelli, M. Dondi, G. Guarini, V. Matteucci, D. Biasini, D. Gardini, Ceram. Ind. 11, 3 (2006) 7-12.

[7] L. C. R. Carpinetti, E. V. Gonçalves F., A. J. V. Porto, C. R. G. Jasinevicius, Rugosidade Superficial - Conceitos e Princípios de Medição, Serviço Gráfico USP - EESC, S. Carlos, SP (1996) 6.

[8] B. S. Parra, R. C. Gennari, F. G. Melchiades, A. O. Boschi, Ceram. Ind. 11, 2 (2006) 15-18.

[9] R. Bernardes Filho, L. H. C. Mattoso, Microscopia de Força Atômica, in S. V. Canevarolo Jr. (Ed.), Técnicas de Caracterização de Polímeros, Artliber, S. Paulo, SP (2003) 201-207.

[10] F. C. Duarte, Microscópio de Tunelamento com Varredura (STM) e Microscópio de Força Atômica (AFM), disponível em http://www.dsif.fee.unicamp.br/, 04/01/2010. [11] G. Kigle-Boecker, Metal Finishing 93, 5 (1995) 28-31. [12] K. Boussu, B. Van der Bruggen, A. Volodin, J. Snauwaert, C. Van Haesendonck, C. Vandecasteele, J. Colloid Int. Sci. 286 (2005) 632-638.

(Rec. 31/03/2010, Ac. 28/05/2010) 